\title{
Norois
}

Environnement, aménagement, société

202 | 2007/1

Recomposition des espaces ruraux

\section{Refaire campagne en Île-de-France}

Making the countryside anew in Île-de-France

\section{Monique Poulot et Thérèse Rouyres}

\section{(2) OpenEdition}

Journals

Édition électronique

URL : https://journals.openedition.org/norois/1631

DOI : $10.4000 /$ norois. 1631

ISBN : 978-2-7535-1550-5

ISSN : 1760-8546

\section{Éditeur}

Presses universitaires de Rennes

Édition imprimée

Date de publication : 1 mars 2007

Pagination : 61-71

ISBN : 978-2-7535-0457-8

ISSN : 0029-182X

\section{Référence électronique}

Monique Poulot et Thérèse Rouyres, «Refaire campagne en Île-de-France », Norois [En ligne], 202 |

2007/1, mis en ligne le 01 mars 2009, consulté le 13 janvier 2022. URL : http://

journals.openedition.org/norois/1631 ; DOI : https://doi.org/10.4000/norois.1631

(c) Tous droits réservés 


\title{
Refaire CAMPagne en Île-De-France
}

\author{
Monique Poulot, Thérèse Rouyres \\ GECKO - EA 375 \\ (Université Paris X - Nanterre), \\ 200 avenue de la République - 92001 NANTERRE cedex \\ ppoulot@club-internet.fr, trouyres@libertysurf.fr
}

\begin{abstract}
RÉSUMÉ
Longtemps laissée aux seuls agriculteurs, la campagne est désormais revendiquée par les citadins et les accourus comme un cadre de vie paysager, notamment en Île-de-France, première région urbaine où les espaces ouverts couvrent encore $80 \%$ des superficies. Repenser ces campagnes, voire en refaire, suppose de concilier les attentes d'agriculteurs, peu nombreux mais ultra performants et gérant des coupures vertes, avec celles des nouveaux habitants, tout en restaurant la qualité de l'environnement, mis à mal autant par l'urbanisation rapide que par les pratiques agricoles.
\end{abstract}

Mots CLÉS : Aménagement rural-Aménités rurales - Campagne-nature-Campagnes périurbaines - Multifonctionnalité de l'agriculture.

\section{ABSTRACT}

\section{Making the countryside anew in Île-de-France}

The countryside, only devoted to the farmers during the $20^{\text {th }}$ century, is nowadays claimed by city dwellers and the new inhabitants of peri-urban areas as a landscaped environment, especially in Île-de-France where open spaces still represent $80 \%$ of the region. In these areas, it's necessary to reconcile the efficient farmers' expectations with the new inhabitants' ones. This goal has to be achieved while restauring the landscape damaged by the rapid urbanization as well as the agricultural techniques. It means here "to rebuild the countryside".

KEY WORDS : Agricultural Multifunctionality - Countryside - Peri-urban Areas - Rural Amenities - Rural Planning.

La majorité des Français se représente l'Île-de-France comme une région très fortement urbanisée et les Franciliens eux-mêmes estiment à $40 \%$ la surface occupée dans leur région par la ville et ses infrastructures ${ }^{1}$ (Houot, 2003; Rosset, 2003). Cette représentation prend acte de 50 ans de développement urbain : de 1968 à nos jours, l'espace urbanisé a doublé, la région a gagné

1. Enquêtes de perception réalisées par des étudiants de l'Université de Nanterre à la demande de l'Institut d'Aménagement et d'Urbanisme de la Région Île-de-France (IAURIF) pour la production d'un Atlas rural et agricole de l'île-de-France. Les analyses de perception sont tirées des deux mémoires cités. 
1,7 million d'habitants avec un fort redéploiement interne puisque les espaces situés au-delà de $30 \mathrm{~km}$ de Notre-Dame abritent désormais $44 \%$ de la population régionale contre $30 \%$ en 1968 (fig. 1). La perception d'intense urbanisation est relayée par l'INSEE qui, dans son zonage en aire urbaine, classe 1211 communes sur les 1300 de la région dans la catégorie « espace à dominante urbaine ».

Dans la réalité, les espaces agricoles et naturels couvrent $80 \%$ de la superficie régionale dont $53 \%$ en cultures, $23 \%$ en forêts et le reste en parcs et jardins (fig. 2). Ces campagnes en rétraction demeurent l'objet de multiples convoitises tant d'une grande culture en quête d'espace que d'une urbanisation où triomphe le modèle pavillonnaire (Poulot et Rouyres, 2001). Les concurrences sont surtout d'ordre foncier mais elles révèlent aussi des logiques fonctionnelles et de vie très différentes selon les habitants-usagers de l'espace rural. Les nouveaux habitants souhaitent vivre dans une campagne-cadre de vie, "paysagée », très éloignée de l'espace de production agricole intensif que veulent conserver les agriculteurs. Tous utilisent pourtant le même vocable « campagne »; tous ont de multiples projets pour l'aménager et la conformer à leurs attentes. Les accourus entendent refaire et protéger la campagne qu'ils ont idéalisée, quand les agriculteurs, soucieux de retrouver une image d'hommes de la terre après les crises alimentaires des dernières années, tentent de redessiner un nouveau cadre pour leur espace de travail intégrant la demande des nouveaux habitants.

Au moment où l'ensemble de la société est amené à « repenser les campagnes » (Perrier-Cornet, 2002), cette démarche prend une signification particulière dans la première région périurbaine de France massivement investie par de nouveaux habitants venus chercher à et dans la campagne « un antidote fantasmatique idéal à la jungle des villes » (Hervieu et Viard, 2001a). Où, comment et pour qui « refait-on campagne »? Quelle part les pouvoirs publics et les associations prennent-ils à cette reconquête? Quels sont les contours de cette ruralité en gestation tant dans les périmètres décrétés que dans les espaces ouverts ordinaires?

\section{La campagne des uns et la campagne des autres}

Les campagnes franciliennes ont connu, avec de simples décalages dans le temps, des évolutions démographiques comparables à celles de tout l'espace rural français. L'exode rural n'y a laissé, dès l'entre-deux-guerres, qu'une majorité d'agriculteurs investis par la III ${ }^{\mathrm{e}}$ République, de « la responsabilité de l'ensemble de l'espace non bâti » (Hervieu et Viard, 2001b). La révolution agricole d'après les années 1950 renforce leur capacité à modeler cet espace conformément à leurs besoins et leur apporte une légitimité économique inédite : porteur d'innovation et de modernité, ce groupe de grands fermiers participe à l'essor de la production qui amène le complexe agro-alimentaire français d'abord à l'autosuffisance puis à l'exportation. La périurbanisation qui s'accélère à partir des années 1960 modifie la donne : les campagnes quasi exclusivement agricoles depuis un siècle reçoivent de nouvelles fonctions, résidentielles et de loisirs, et le mot s'enrichit des représentations que ses nouveaux habitants lui accordent (Mathieu, 1990 et 1998).

\section{LA CAMPAGNE DES « HOMMES DE LA TERRE »}

Région agricole prospère, l'Île-de-France a toujours porté des campagnes variées mettant à profit des terroirs opposant vallées et plateaux. Les premières ont accueilli des activités d'élevage et une ceinture maraîchère et fruitière destructurées par l'essor du transport routier qui cloisonne l'espace et aiguise les concurrences avec d'autres régions (Phlipponeau, 1956; Poulot et Rouyres 2000a). Les seconds ont été dévolus aux céréales dans le cadre de vastes exploitations fermières formées sur les grandes propriétés nombreuses dans une région capitale depuis un millénaire (Brunet, 1960). Ces contrastes de mise en valeur liés autant à la nature qu'à l'histoire sont inscrits dans les modes d'habiter : gros villages d'agriculteurs spécialisés des vallées et grandes fermes à cour fermée sur les plateaux. Aujourd'hui de nombreux agriculteurs ont déserté les villages, remplacés 
par d'autres catégories socio-professionnelles, mais ceux qui restent ont conscience d'appartenir à une vieille lignée d'occupants de cette terre et identifient l'histoire du territoire à celle de leur famille. Cet attachement au lieu est si fort qu'ils le revendiquent souvent en premier avant même leur profession.

L'adoption précoce de méthodes productivistes avec de multiples remembrements ont modelé des paysages aux vastes horizons dénudés : les parcelles atteignent fréquemment 80 à 100 ha et leur trame ne respecte plus les déformations naturelles du terrain; chemins, haies, boqueteaux qui structuraient l'espace et arrêtaient le regard ont disparu. Cette simplification du paysage accompagne la spécialisation dans la grande culture peu exigeante en main-d'œuvre (une UTA pour 150-160 ha) qui gagne les versants de vallées et constitue actuellement les quatre cinquièmes du potentiel agricole régional (en marge brute standard). L'uniformisation des paysages dominés par les terres labourables (96\% de la SAU en 2000) est renforcée par le retournement de prairies désormais drainées et l'abandon de l'élevage bovin et ovin : le blé, cultivé dans les trois quarts des exploitations occupe $44 \%$ de la SAU en 2000 contre $33 \%$ en 1970. La PAC de 1957 et la périurbanisation font que le champ de blé, solution d'attente la plus rentable et forme de culture la moins sensible aux dégradations des nouveaux habitants, s'insinue jusqu'au pied des immeubles.

Les agriculteurs de l'Île-de-France, céréaliculteurs en tête, jouissent d'une grande puissance économique et sont fortement insérés dans des filières internationalisées mais ils sont devenus ultra-minoritaires. En 2000, les 6400 exploitants et les 17000 membres de leur famille et salariés pèsent bien peu face aux 11 millions d'habitants de la région et le nombre d'élus municipaux issus de la sphère agricole est en régression. Leur lien à la région s'est distendu avec le recul du maraîchage (asperges d'Argenteuil, cerises de Montmorency, haricots d'Arpajon...) et la disparition d'établissements de transformation de produits locaux chassés par les progrès de l'urbanisation et les évolutions industrielles (Poulot et Rouyres, 2004) : leur production globale n'assure que $20 \%$ de la nourriture consommée localement. Ainsi la relation de la profession à la terre a changé : le paysan est devenu exploitant ; l'agriculteur nourricier s'est mué en industriel de la terre menaçant les équilibres naturels par une artificialisation excessive des milieux. Cette " figure de la nature » est désormais refusée par la société civile, notamment les périurbains, influencés par la pensée écologique.

\section{DES CAMPAGNES À HABITER ET À RÊVER}

L'essor de l'agglomération parisienne et de la périurbanisation à partir des années 1960 transforme les usages de la campagne. Les deux tiers des arrivants ont quitté une ville à la recherche d'un environnement plus conforme à leurs attentes et quelque $60 \%$ désirent renouer avec des attaches rurales ou des pratiques de vacances. Pour eux, la « campagne », si polysémique soit-elle, se définit surtout par opposition à la ville. Espace de résidence récemment investi, dissocié des lieux de travail et que l'on croit à l'abri des pollutions urbaines, elle figure le lieu de repos du soir et du week-end dans des villages «à taille humaine » empreints de convivialité : le cadre de vie idéal de la résidence secondaire est devenu celui de la résidence principale.

Cette campagne se reconnaît par « ses arbres, sa verdure, ses champs, ses bois ». Pour 70 \% des Franciliens, elle est un décor où l'agriculture n'apparaît qu'en filigrane, un paysage appréhendé par les sens et non un lieu de production. C'est là le divorce majeur entre les anciens usagers de cet espace et les nouveaux arrivés. La campagne se définit aussi par ses opportunités de loisirs dans des espaces ouverts et « vides » qui donnent une sensation de liberté. Ignorant tout du fonctionnement de l'agriculture, les accourus considèrent comme un bien commun non approprié où tout est permis. C'est un autre point de friction avec la campagne des agriculteurs.

Plus de $90 \%$ des périurbains apprécient leur lieu d'habitation qui conjugue proximité de la ville et qualité de vie à la campagne. Cette dernière renvoie aux aspects paysagers et écologiques qui lui sont attribués et à des constructions imaginaires se référant à l'enfance et au passé. Dans cette quête nostalgique, vivre à la campagne est perçu comme un moyen de retrouver du naturel, de 


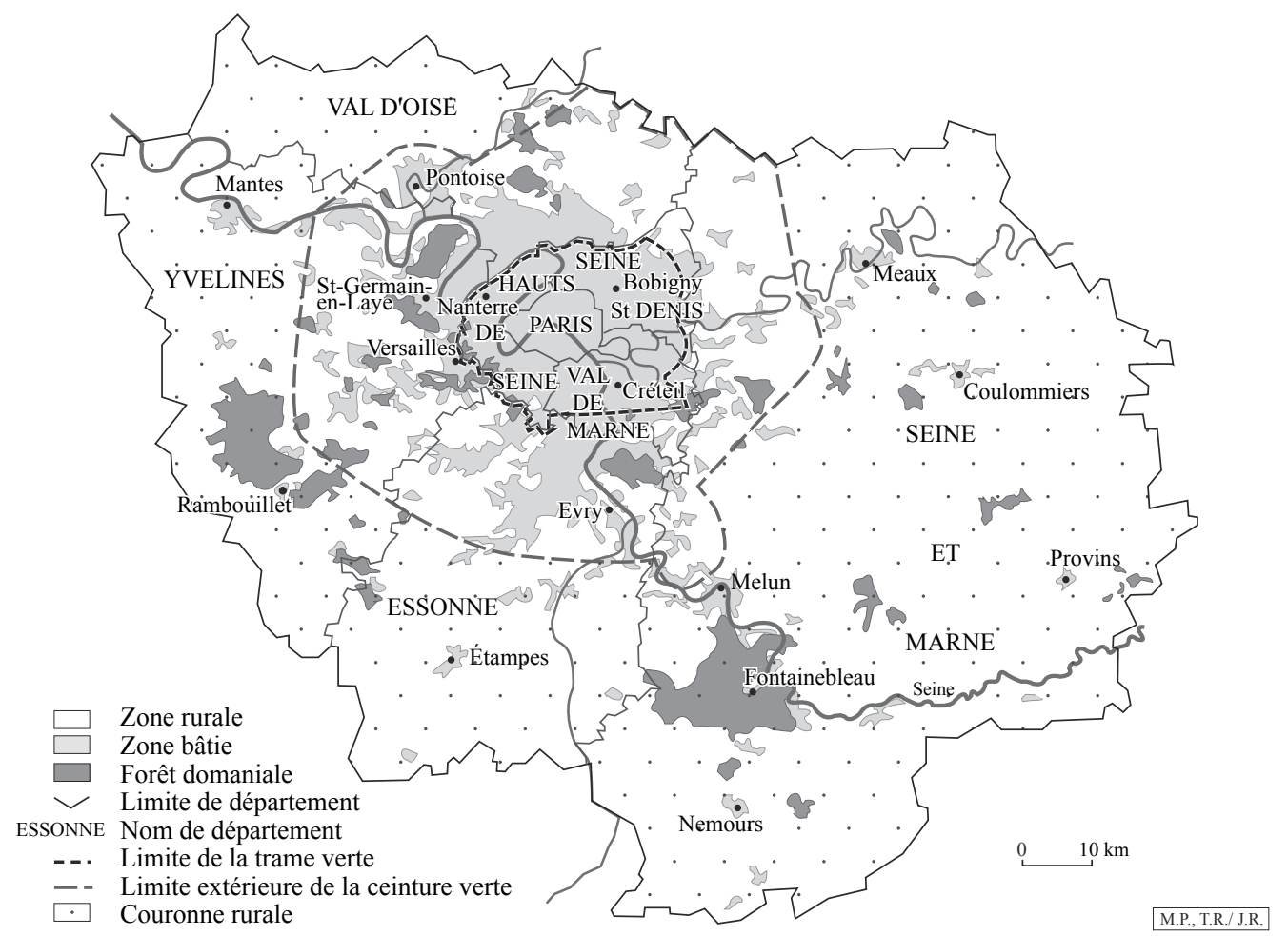

Figure 1 : L'île-de-France, découpages administratifs et découpages paysagers Administrative and landscaped divisions in Île de France

l'authentique voire de «vraies valeurs » que la ville a perverties. Cette campagne-mémoire qui relève de l'image d'Épinal est jardinée, sans odeurs, sans bruits, presque sans agriculture; elle évoque les tableaux bucoliques de l'agriculture traditionnelle. Cette campagne fantasmée participe ainsi de la vague générale de patrimonialisation qui touche actuellement toute la société française (Chevallier, 2000). Le " patrimoine rural » s'étend désormais à la nature, aux paysages, au passé, au calme et à l'espace. La crainte de voir ce lieu, choisi pour ces aménités, dénaturé conduit nombre de nouveaux habitants à former des associations de défense environnementales $(300$ avec 30000 adhérents) afin de limiter l'urbanisation et préserver la campagne dans un réflexe NIMBY2 ${ }^{2}$ Les enjeux paysagers et urbanistiques ont été au cœur des dernières élections municipales de 2001 avec la victoire d'équipes sensibilisées à ces questions.

Anciens et nouveaux habitants de la campagne francilienne partagent désormais un espace qu'ils perçoivent et vivent de manière différente, souvent contradictoire. Si les agriculteurs, chefs d'entreprise soumis à des contraintes économiques mais aussi mémoire des lieux et gestionnaires de l'espace, représentent la figure de la «campagne-ressource », les accourus plébiscitent une « campagne paysage et cadre de vie » dotée d'une valeur immatérielle. Ces deux approches vigoureusement concurrentes doivent intégrer la vision des campagnes occidentales proposée par les scientifiques soucieux de la durabilité : la «campagne nature » respectueuse de la biodiversité et des grands équilibres naturels ${ }^{3}$ (Perrier-Cornet, 2002). Le modèle des campagnes de la deuxième

2. L'effet NIMBY (Not in my back yard) qualifie les discours et les pratiques d'opposition de populations à l'implantation de nouveaux logements et lotissements, surtout s'ils sont porteurs de mixité sociale.

3. Définitions du groupe de prospective sur les espaces naturels et ruraux de la DATAR (DIACT). 
révolution agricole est obsolète; pour rester agriculteur aujourd'hui, « le paysan doit d'une certaine manière entrer dans le paysage pensé de la ville et dans une nouvelle étape du savoir de nature » (Hervieu et Viard, 2001b)

\section{Les gestes du refaire campagne}

Les Franciliens sont tous concernés par les trois visages de leurs campagnes avec des degrés d'implication divers mais le vivre ensemble exige des rencontres : la campagne-production s'enrichit de la demande de nouvelles prestations des périurbains tandis que la campagne-paysage ne peut se construire sans l'agriculture et les agriculteurs. Enfin, tous, dans leur volonté de « refaire campagne », doivent répondre aux sollicitations de la société civile qui pour faire contrepoids à une anthropomorphisation jugée excessive, voire dangereuse de l'espace rural, réclame de lui réserver une part de naturel.

\section{REDESSINER UN CADRE DE TRAVAIL}

Comme dans toutes les régions périurbaines, la sécurisation du foncier agricole s'avère le préalable à toute réorganisation de l'espace de production. Longtemps considéré comme une simple réserve foncière bon marché, l'espace rural francilien ne conquiert son existence qu'avec la publication en 1976 du Schéma directeur d'aménagement et d'urbanisme (SDAU) de la région Ile-de-France alors que le processus d'urbanisation est déjà très avancé et que la décentralisation sensibilise l'opinion régionale au besoin de nature dans la ville élargie. Mais les quelques outils (procédure d'Espaces naturels sensibles et préemption par l'Agence des espaces verts ou AEV fondée en 1976) sont surtout utilisés alors pour préserver la forêt.

La décennie 1990 amorce le temps des décisions; le Plan vert adopté en 1994 propose un zonage paysager et environnemental inscrit dans le Schéma directeur de la région Île-de-France (SDRIF) de la même date (fig. 1). Les espaces ouverts franciliens y sont classés en deux catégories : la ceinture verte entre 10 et $30 \mathrm{~km}$ de Notre-Dame avec 60000 ha de cultures enclavés et hachés par l'habitat et les infrastructures; au-delà la couronne rurale qui demeure le principal espace agricole avec 530000 ha, notamment en Seine-et-Marne. Le SDRIF de 1994 préconise la « reconstruction de la ville sur la ville » afin «d'épargner la ceinture verte » et « d'économiser l'espace rural dans la couronne rurale ». Il s'agit de bâtir dans la continuité des noyaux existants avec une densité de constructions décroissante vers la périphérie sans consommer plus de 1750 ha par an (près de quatre fois moins que durant la période d'extension maximale des années 1968-1977). Enfin les collectivités sont invitées à garantir dans leur POS ou SDAU des espaces agricoles cohérents, suffisamment étendus pour assurer des conditions satisfaisantes d'exploitation : 2000 ha en grande culture, 300 en agriculture spécialisée et 50 pour les serres. La contribution de l'Île-de-France au Schéma des services collectifs des espaces naturels et ruraux (SSCENR), prévu par la Loi d'aménagement et de développement durable du territoire (LOADDT) du 25 juin 1999, confirme cette volonté de maintien d'espaces ouverts : la promotion d'une nouvelle urbanité passe par la préservation des espaces agricoles et naturels tant « remarquables » qu'ordinaires ${ }^{4}$ (fig. 2).

Ces préconisations du SDRIF paraissent insuffisantes aux agriculteurs qui ont obtenu par la loi d'orientation agricole de juillet 1999, la possibilité de créer des zones agricoles protégées notamment en milieu périurbain (art. 108 de la LOA). La sécurisation foncière de ces espaces est garantie par leur inscription aux Plans locaux d'urbanisme (PLU) en tant que servitude d'utilité publique et confortée en Île-de-France par l'intervention de la SAFER et de l'AEV; deux expériences sont en cours à Vernouillet et aux Essarts-le-Roi dans les Yvelines (fig. 3). Les instruments de protection de l'espace agricole se sont donc multipliés récemment, à l’image du PLU qui remplace le POS : envi-

4. Préfecture de l'Île-de-France, Schéma des Services Collectifs des Espaces Naturels et Ruraux, novembre 1999, 78 p. 


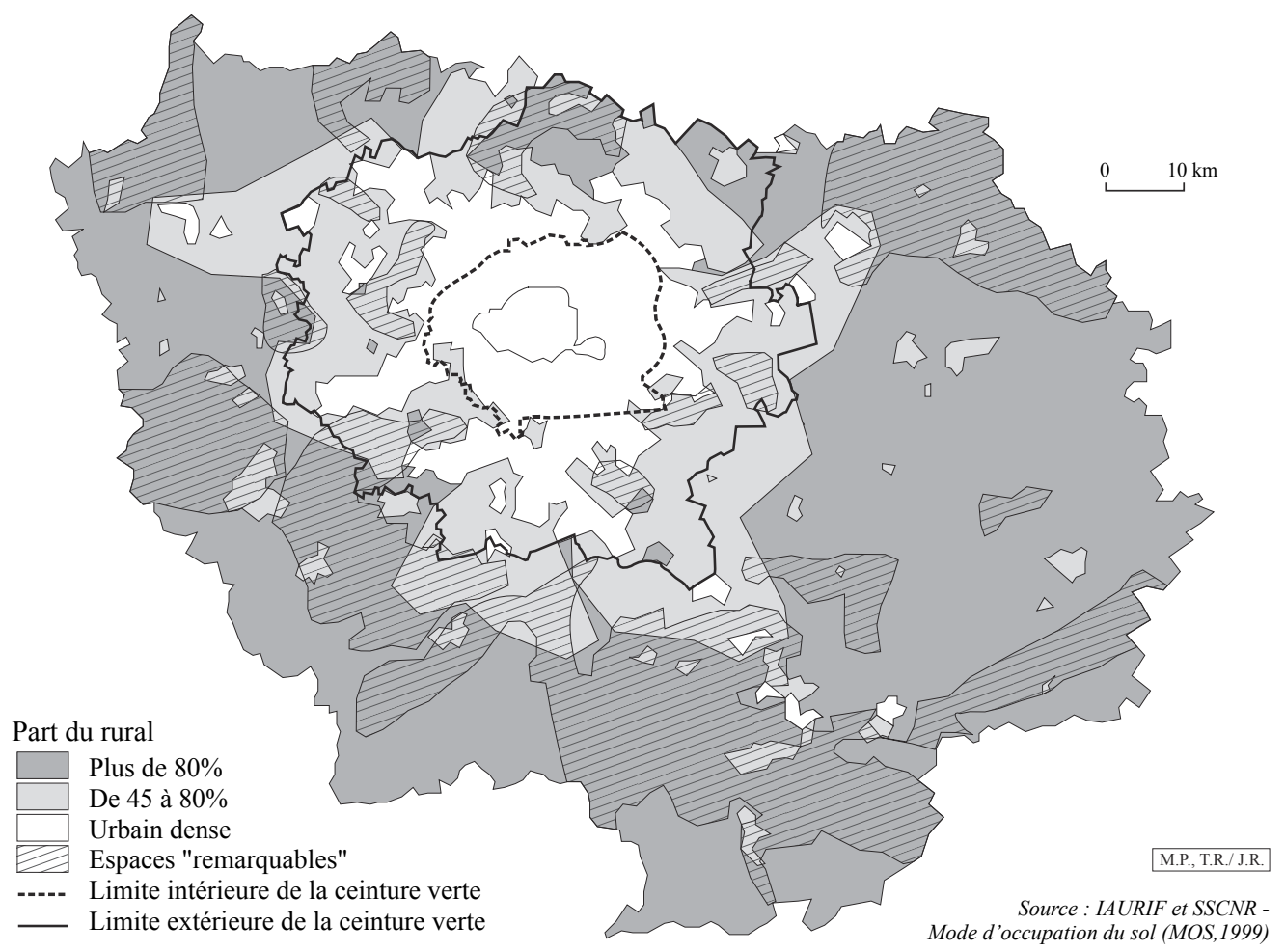

Figure 2 : Les campagnes franciliennes The peri-urban areas in Île de France

sagé à l'échelle intercommunale, il permet de délimiter des espaces de protection suffisamment vastes pour dépasser le seuil critique nécessaire à leur rentabilité (Poulot et Rouyres, 2003).

Mais la sécurité foncière ne suffit pas au maintien d'activités agricoles car les conditions d'exploitation sont rendues plus difficiles ici par la proximité des constructions et des voies de communication. L'éclatement du parcellaire lié à la concentration récente des exploitations allonge les temps de parcours d'engins agricoles qui doivent emprunter des voies de circulation, générale au trafic très dense. De nombreux agriculteurs souhaitent séparer les circulations, y compris sur les chemins d'accès aux parcelles de plus en plus fréquentés pour les loisirs et vecteurs de déprédations. Pour atténuer ces handicaps, des brigades montées de surveillance (Vernouillet) et des indemnités locales ou régionales ont été créées; l'aide régionale à l'horticulture et au maraîchage, instituée en 1990, vient ainsi compenser une partie des surcoûts liés à ces dommages.

Les interventions publiques soulignent les spécificités de l'agriculture autour des agglomérations. Elles sont la reconnaissance, au moment où l'espace périurbain s'impose dans les zonages INSEE, de la lente émergence de l'agriculture éponyme dans une nouvelle campagne s'ouvrant aux souhaits et aux besoins de la société civile urbaine dominante. En effet, si l'expansion urbaine a joué un rôle déstabilisant sur l'agriculture, la présence d'une population nombreuse, à la recherche de «plus de naturel », rend possible des diversifications qu'il eût été difficile de mettre en place auparavant (Poulot et Rouyres, 2000b). Ce mouvement concerne $20 \%$ des exploitations qui fonctionnent comme des laboratoires de l'innovation dans les relations entre ruraux et urbains. La vente directe à la ferme et sur les marchés assure un débouché rémunérateur pour la presque totalité des producteurs d'œufs et de volailles et pour $45 \%$ des maraîchers. Une trentaine de grandes exploitations ont ouvert des activités de cueillette fonctionnant près de neuf mois dans l'année, du temps des 


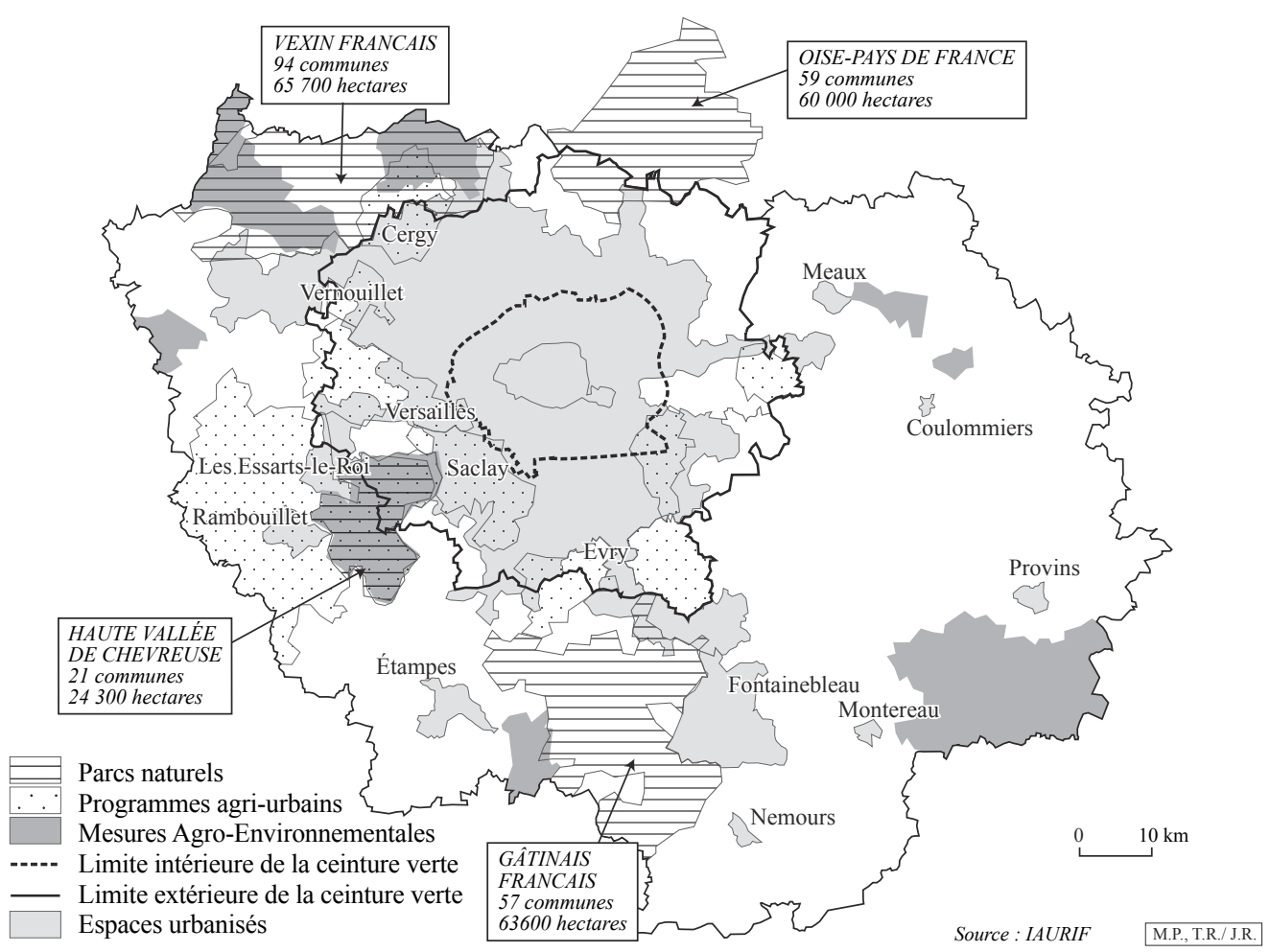

Figure 3 : Les principales démarches pour « refaire campagne » en Île-de-France How to rebuild parisian countryside

tulipes à celui des pommes et, la plupart vendent des produits transformés provenant des fermes voisines (conserves, confitures, sorbets, volailles...). Plus généralement, portés par la demande régionale et les exigences récentes en termes de qualité, les produits de terroir connaissent un certain renouveau depuis les AOC (Brie de Meaux et de Melun en Seine-et-Marne) jusqu'aux labels rouges (volailles de Houdan...). Enfin des filières locales affichant la marque Parc naturel cherchent à séduire le consommateur périurbain avec la menthe et le cresson de fontaine pour le Gâtinais français ou le lapin compote et le jus de pomme pour le Vexin. La création en 2001 du Comité de promotion des produits agricoles et agro-alimentaires de Paris-île-de-France prend acte de la (re)naissance d'une agriculture de proximité.

La plupart des agriculteurs engagés dans cette nouvelle approche de l'agriculture régionale ont également le souci de faire connaître leur métier aux citadins pour renouer des liens distendus. Des «journées portes ouvertes » organisées par filière ou par territoire sont régulièrement proposées. Parfois l'accueil devient un atelier spécifique offrant un contact direct avec la nature et les animaux ainsi que des animations à des publics variés, notamment les enfants des écoles. Les 85 fermes pédagogiques de la région, dont 50 sont de grandes exploitations productives, accueillent chaque année plusieurs milliers d'enfants pour une journée ou une demi-journée. Les autres formes d'accueil - camping à la ferme, gîte, table d'hôtes - restent modestes car peu adaptées à l'organisation du travail en grande culture. Ces démarches qui intègrent les demandes du public font entrer certains agriculteurs franciliens dans une logique de production valorisant le territoire (Bonnerandi et al., 2003). Elles concourent à la création d'une nouvelle campagne périurbaine. 


\section{INVENTER UN DÉCOR DE CAMPAGNE}

Les campagnes franciliennes deviennent multifonctionnelles et doivent emporter l'adhésion de tous leurs habitants : lieux de production pour les agriculteurs, elles sont pour les périurbains des territoires de vie au paysage plaisant à regarder. Ce dernier, standardisé par les évolutions contemporaines de l'agriculture, ne correspond plus aux images et représentations des urbains. Le paysage de la grande culture, représentatif d'une technicité contestée et trop monotone, ne séduit guère en dépit de la sensation d’immensité et de liberté qu’il suggère. La diversité, déjà recherchée par les premiers périurbains qui ont plébiscité les Yvelines aux paysages vallonnés, n’y apparaît que par le jeu des couleurs des cultures, entre les camaïeux de verts au printemps et le jaune éclatant du colza. De la même manière, les paysages liés au maraîchage sont méconnus et rejetés : les serres qui évoquent le bâti industriel, les films plastiques et les amoncellements de cageots en bordure de champs choquent les attentes esthétiques de promeneurs imprégnés de visions bucoliques et pastorales.

Les agriculteurs sont ainsi confrontés à une demande de production immatérielle très subjective faisant référence à un passé imaginé : ils ont à inventer, par l'agriculture, un décor dans lequel les impératifs de production ne seront pas visibles; ils doivent ajouter à leur fonction première l'entretien du «vaste jardin » de la ville. Dans cette nouvelle logique où le paysage devient bien commun de la société et patrimoine à transmettre, sa construction et sa conservation acquièrent une valeur marchande et ses coûts de production appellent rémunération. Longtemps réticents à devenir les « jardiniers de la nature », les agriculteurs y sont désormais contraints par l'ampleur du mouvement sociétal et la nouvelle Politique agricole commune. L'offre de services aux citadins et l'entretien des paysages représentent une alternative pour des exploitants qui perçoivent de moins en moins d'aides à produire et ne peuvent sans subventions maintenir leur revenu ${ }^{5}$. Cette évolution vers une agriculture de services est l'un des scénario envisagés par Philippe Lacombe dans son exploration de L'agriculture à la recherche de ses futurs.

L'Île-de-France apparaît, surtout dans sa partie occidentale, comme le terrain d'élection de cette forme d'agriculture notamment quand elle gère des coupures vertes. La puissance des organisations environnementalistes, l'attitude des équipes municipales engagent les agriculteurs à adhérer à ce schéma qui leur offre une nouvelle légitimité sociale. Des partenariats financés par le Conseil régional et les Conseils généraux aboutissent à la signature de documents contractuels entre les différents acteurs : ces programmes agri-urbains se déclinent en charte de développement agricole comme à Vernouillet ou en chartes patrimoniales à Saclay, Versailles et dans la plaine de Cergy... (fig. 3) (Poulot, 2006). Les préconisations incitent à la remise en culture de parcelles abandonnées, à la reconstitution de haies aux espèces variées et de bandes enherbées le long des rivières. Il s'agit d'améliorer, voire réhabiliter, les transitions entre espaces agricole, urbain et boisé pour assurer une continuité visuelle et biologique, de rechercher des aménagements paysagers mettant en scène l'espace agricole et son bâti en soignant les abords de ferme et en valorisant le bâti de qualité. La réalisation de cette nouvelle campagne échoit aux seuls agriculteurs, parfois conseillés par des paysagistes, pour ménager des points de vue aux périurbains alors que dans le même temps peu de contraintes pèsent sur les caractéristiques du bâti pavillonnaire et de ses jardins uniformément clos de haies de thuyas. L'agriculture devient un faire-valoir des espaces résidentiels, volontiers érigée par les associations périurbaines en alibi pour maintenir des espaces ouverts et contenir l'urbanisation.

Ces deux figures de reconstruction, celle de campagne-décor comme celle de campagne périurbaine, illustrent le vécu de chacun des groupes partageant l'espace périurbain sans véritablement résoudre leurs antagonismes. Elles peinent plus encore à renouer avec l'idée de nature dans une logique de durabilité.

5. Le Monde, 24 février 2004 : «Que faut-il faire des agriculteurs français? », supplément Économie. 


\section{REDONNER SA PLACE À LA NATURE}

L'environnement en Île-de-France a été soumis à de nombreux stress qui ont des effets destructeurs tant sur la qualité de l'air que sur celle des eaux. La responsabilité en incombe aussi bien aux méthodes de production agricole qu'aux conséquences d'une urbanisation rapide multipliant les pollutions de toute nature. Deux tiers des captages pour l'alimentation en eau potable affichent des teneurs en nitrates supérieures à $20 \mu \mathrm{g}$ avec des pics à plus de $50 \mu \mathrm{g}$ en Beauce et seulement $17 \%$ fournissent une eau proche de l'état naturel si bien que la totalité de la région est classée en zone vulnérable. Les nappes de Champigny en Brie centrale et celle de la vallée de la Seine-aval révèlent des concentrations en produits phytosanitaires très largement supérieures à la moyenne admise dans les directives européennes.

L'opinion est de plus en plus sensibilisée aux effets néfastes pour la santé de ces agressions avec une conscience plus aiguë de la participation des agriculteurs que de celle des urbains pourtant grands pourvoyeurs d'oxyde de carbone ou jardiniers amateurs à la main lourde ${ }^{6}$. La restauration des équilibres naturels passe par la mise en place de réglementations, notamment dans le cadre de la PAC, qui incitent les agriculteurs à produire autrement. Les premières mesures n'ont guère eu d'écho en Île-de-France à l'exception des Mesures agri-environnementales (MAE) «Opérations locales de maintien et de gestion extensive des prairies humides » dans les Parcs naturels régionaux du Vexin et de Chevreuse et d'un Plan de développement durable à Rambouillet (Charvet et al., 1998) (fig. 3). Quant aux Contrats territoriaux d'exploitation (CTE) de la Loi d'orientation agricole de 1999 devenus Contrats d'agriculture durable, ils n'ont séduit que 271 exploitations avec un volet environnemental allégé centré sur la gestion des intrants et le travail simplifié du sol (chiffres 2002). Enfin, seules deux opérations ont obtenu le label «Ferti-mieux » en Seine-et-Marne.

Au vu de ces résultats, redonner une place à la nature n'est pas le souci des agriculteurs franciliens qui peinent à changer leurs manières de faire; leur faible implication dans l'agriculture biologique, alors qu'ils disposent du plus grand marché de France, en est une autre illustration (64 agriculteurs majoritairement céréaliers en 2001 sur 2667 ha). La création de quatre Parcs naturels régionaux depuis 1985 apparaît comme la principale démarche de mise en nature dans l'espace francilien; sur $13 \%$ de la superficie régionale, ils concentrent l'essentiel des opérations depuis les chartes paysagères dans le Vexin jusqu'à la protection des milieux humides et l'affichage de signes de qualité dans le cadre d'opérations locales. Encore faut-il rappeler qu'ils se sont construits tout autant contre l'avancée urbaine, notamment des villes nouvelles de Saint-Quentin-en-Yvelines, Cergy et Évry, que dans une optique environnementale comme le rappelle leur charte. Au total, la reconquête d'espaces plus naturels se limite aux périmètres décrétés; ailleurs les logiques productivistes s'infléchissent lentement vu le type d'agriculture dominant. Les accords de Luxembourg de juin 2003 mettent pourtant la campagne-nature au cœur de la PAC puisque, depuis 2005, les aides directes sont liées au respect de directives portant sur la préservation de la nature, la qualité de l'eau, le bien-être animal et les recommandations de Natura 2000.

\section{Conclusion}

Repenser la campagne et la refaire est un thème qui traverse toute la société de l'Europe occidentale au moment où une majorité de la population souhaite bénéficier des aménités rurales avec les avantages de la vie en ville. Lîle-de-France participe pleinement de ce projet de « villecampagne » si bien que la maîtrise du développement urbain et la valorisation de la campagne s'y affirment désormais comme deux volets complémentaires d'une même politique d'aménagement. L'espace rural y est devenu le cadre paysager de la ville tout en demeurant un espace de production puissant et la campagne de demain se doit de répondre à toutes ces attentes. L'évolution vers une

6. Leur consommation de phytosanitaires représente $10 \%$ de celle du secteur agricole sur $2 \%$ de l'espace régional; et que dire de la consommation en herbicides de la SNCF... 
agriculture périurbaine paraît la plus simple à conduire; de tout temps les agriculteurs se sont adaptés et ont su innover pour satisfaire de nouvelles demandes : les campagnes périurbaines ne sont qu'un stade dans le temps long de l'agriculture. L'accès à la durabilité est déjà plus complexe à mettre en place non pas tant pour des raisons techniques plutôt bien maîtrisées que pour les changements nécessaires de manières de faire : les céréaliers y voient un alourdissement de leurs charges de travail et des risques accrus d'accidents de culture sans y trouver de rémunération compensatrice. Ces campagnes décrétées se multiplient néanmoins sous la pression de la société civile. Le problème financier reste aussi au cœur de la construction de la campagne-décor des citadins; plus fondée sur l'art des jardins que sur des logiques agricoles est-elle encore de la responsabilité des seuls agriculteurs? N'est-elle pas de toute façon une « autre fin des paysans? »

\section{Bibliographie}

Allie (L.), Briant (C.), 2003. - « Les Parcs Naturels Régionaux français : un modèle de gouvernance et de planification spatiale pour le milieu périurbain? ", Canadian Journal of Science/Revue canadienne des Sciences Régionales, $n^{\circ}$ spécial : «Structures et dynamiques au-delà des banlieues », vol XXVI, 2 et 3, p. 447-463.

Berger (M.), 2004. - Les périurbains de Paris. De la ville dense à la métropole éclatée?, Paris, CNRS, $320 \mathrm{p}$.

Bonnerandi (E.), Landel (P.-A.), Roux (E.), 2003. - « Les espaces intermédiaires, forme hybride : ville en campagne, campagne en ville », Revue de géographie alpine, n spécial : « les agriculteurs dans la cité », T. 91, n 4, p. 67-79.

BRunet (P.), 1960. - Structure agraire et économie rurale des plateaux tertiaires entre la Seine et l'Oise, Caen, Caron et Cie, 552 p.

Charvet (J.-P.), 1985. - Les greniers du monde, Paris, Economica, 368 p.

—, 2003. - «Les conditions du maintien d'une agriculture vivante », Canadian Journal of Science/Revue canadienne des Sciences Régionales, ${ }^{\circ}$ spécial : Structures et dynamiques au-delà des banlieues, vol XXVI, 2 et 3, p. 359-371.

Charvet (J.-P.), Poulot (M.), Rouyres (T.), 1998. - « Les Plans de développement durable dans les régions de grande culture du Bassin parisien », dans Croix (N.) (dir.), Environnement et nature dans les campagnes, Rennes, PUR, p. 153-167.

Chevallier (D.) (dir.), 2000. - « Vives campagnes, le patrimoine rural, projet de société », Autrement, n 194, $223 \mathrm{p}$.

Donadieu (P.), Fleury (A.), 2003. - « La construction contemporaine de la ville-campagne en Europe », Revue de géographie alpine, $\mathrm{n}^{\circ}$ spécial : les agriculteurs dans la cité, T. 91, nº 4, p. 19-29.

DRIAF-IAURIF, 2004. - Atlas Rural et Agricole de l'île-de-France, 180 p.

Hervieu (B.), Viard (J.), 2001 a. - Au bonheur des campagnes, La Tour-d'Aigues, L'Aube, 160 p.

—, 2001 b. - L'archipel paysan, la fin de la république agricole, La Tour-d'Aigues, L'Aube, 124 p.

Houot (A.), 2003, Perception de l'espace rural par les périurbains de l'ouest francilien, Mémoire de maitrise, Université Paris X-Nanterre, $178 \mathrm{p}$.

LACOmbe (P.) (dir.), 2002. - L'agriculture à la recherche de ses futurs, La Tour-d'Aigues, L'Aube/DATAR, $192 \mathrm{p}$.

Mathieu (N.), 1990. - « La notion de rural et les rapports ville-campagne en France. Des années 50 aux années 80 », Économie rurale, $n^{\circ} 197$, p. 35-41.

—, 1998. - « La notion de rural et les rapports ville-campagne en France. Les années 90 », Economie rurale, $n^{\circ} 247$, p. 11-20.

Perrier-Cornet (P.), 2002. - Repenser les campagnes, La Tour-d'Aigues, L'Aube/DATAR, 280 p.

Phlipponneau (M.), 1956. - La vie rurale de la banlieue parisienne, étude de géographie humaine, Paris, Armand Colin, 597 p. 
Poulot (M.), 2006. - « Les programmes agri-urbains en Île-de-France : de la «fabrique » de territoires périurbains », communication au colloque «Les dynamiques des territoires en milieu périurbain », Montréal, avril 2006, [vrm.ca/périurbain.asp], octobre 2006, 14 p.

Poulot (M.), Rouyres (T.), 2000a. - « La ceinture maraîchère et horticole francilienne entre production économique et production de paysage », Méditerranée, n 3-4, p. 51-58.

—, 2000b. - « La difficile évolution des campagnes franciliennes vers un espace rural multifonctionnel », Hommes et Terres du Nord, $\mathrm{n}^{\circ}$ 4, p. 253-260.

—, 2001. - « Du rôle patrimonial de l'espace agricole en Île-de-France », dans BERGER (A.) (dir.), Dynamique rurale, environnement et stratégies spatiales, Montpellier, CNRS/Université Paul-Valéry, p. 189-198.

—, 2003. - «Les espaces ouverts en Île-de-France : quels enjeux pour quels acteurs? », Canadian Journal of Science/Revue canadienne des Sciences Régionales, $n^{\circ}$ spécial : Structures et dynamiques au-delà des banlieues, vol XXVI, 2 et 3, p. 431-446.

—, 2004. - « L'Île-de-France agro-alimentaire : vers de nouvelles relations territoriales entre IAA et agriculture? », dans MargetiC (C.), Dynamiques agro-industrielles et dynamiques rurales, Arras, Artois Presses Université, coll. " Géographie », p. 109-131.

Rosset (E.), 2003. - Perception de l'espace rural par les périurbains de l'est francilien, Mémoire de maîtrise, Université de Paris X-Nanterre, 125 p.

ROUYREs (T.), 1994. - « La cueillette à la ferme : diversification ou nouvelle activité dans les zones périurbaines. L'exemple de L'Île-de-France », BAGF, n² 2, p. 216-222.

Cet article a été reçu le 30 juin 2006 et définitivement accepté le 15 janvier 2007. 
